\title{
Validation of the Ottawa Ankle Rules in Indian Scenario
}

\author{
Sanjay Meena ${ }^{1, *}$; Shreesh Kumar Gangary ${ }^{1}$ \\ ${ }^{1}$ Department of Orthopedics, Maulana Azad Medical College, New Delhi, India \\ *Corresponding author: Sanjay Meena, Department of Orthopedics, Maulana Azad Medical College, New Delhi, India. Tel: +91-9968444612, E-mail: sanjaymeena@hotmail.com
}

Received: June 5, 2014; Accepted: February 23, 2015

\begin{abstract}
Background: Ankle injuries are one of the most common presentations in emergency department. Ottawa Ankle Rules (OARs) have been used to predict the requirement of radiographs.

Objectives: This study aimed to validate the OARs protocol for predicting ankle and midfoot fractures in Indian population.

Patients and Methods: A prospective study was conducted in a teaching hospital in north India, during a period of nine months, including all patients who presented with complaints in the ankle region and evolution of less than 48 hours. The study excluded patients with multiple trauma and Glasgow coma scale of less than 15 . All patients underwent clinical evaluation, followed by radiographs depending upon the location of the complaints. Radiographic study results were evaluated by orthopedic surgeons who had not seen the patient.

Results: We evaluated 140 patients ( 84 males and 60 females) with the mean age of 35.2 (range, 8 - 76 years). Of the 140 evaluable patients, 71 had positive criteria for radiological evaluation of which 43 presented with fracture, 69 had negative criteria for radiography with no fracture. The sensitivity of OARs to detect fractures was $100 \%$. The implementation of the OARs appears to have the potential to reduce the number of radiographs for the assessment of these patients by about $51 \%$.

Conclusions: The implementations of OARs have the potential to reduce the number of X-ray graphics needed to assess these patients by about 51\%. The results of this study demonstrate no false negatives and are in agreement with results from other similar studies. It encourages us to implement these criteria in our services urgently, with all the resulting socio-economic implications.
\end{abstract}

Keywords: Ottawa Ankle Rules; Injury; Fracture

\section{Background}

Injury of the ankle joint is common with an annual incidence of ankle fractures is 122 per 100000 people (1). The acute trauma of this joint is one of the main reasons for visits to Emergency Department (ED). It is estimated that only about $15 \%$ of these patients have fracture (2). In most hospitals, it is a common practice to request radiographs of ankle and/or foot although $85 \%$ of the examinations prove negative for presence of fractures (2). To reduce the number of unnecessary ankle and foot radiographs, Stiell et al. from Canada developed a set of rules for obtaining radiographs called Ottawa Ankle Rules (OARs) (3). Ottawa ankle rules are based on the objective criteria that reduce the subjective component of the clinical evaluation, providing specific indications for radiographs and allowing reduction in hospital costs, the exposure to ionizing radiation, and the waiting time in emergencies. According to these guidelines, radiographs of the ankle joint are recommended only if there is any pain in the malleolar zone and any one of the followings: bone tenderness along the distal $6 \mathrm{~cm}$ of the posterior edge of the tibia or tip of the medial malleolus; bone tenderness along the distal $6 \mathrm{~cm}$ of the posterior edge of the fibula or tip of the lateral malleolus; or an inability to bear weight both immediately and in the ED for four steps.

The Ottawa foot rules are for assessing whether a foot
$\mathrm{X}$-ray series is indicated. It states that they are indicated if there is any pain in the midfoot zone and any one of the followings: bone tenderness at the base of the fifth metatarsal (for foot injuries); bone tenderness at the navicular bone (for foot injuries); or an inability to bear weight both immediately and in the ED for four steps

\section{Objectives}

Several attempts have been made to validate the OARs in different countries. Our goal for this study is to provide the evidence for the use of the OARs as a method for the prediction of significant fractures in an Indian clinical setting.

\section{Patients and Methods}

This prospective study was conducted in the ED of a teaching hospital in North India over the nine months from February to November 2011. We included all patients who had sustained ankle and/or midfoot injury with less than 24 hours of injury. All patients were clinically examined by an orthopedic surgeon. We excluded patients with multiple trauma, pregnant woman, patients with altered level of consciousness (Glasgow coma scale < 15), or those who returned to ED for reassessment of the

Copyright (C) 2015, Kashan University of Medical Sciences. This is an open-access article distributed under the terms of the Creative Commons Attribution-NonCommercial 4.0 International License (http://creativecommons.org/licenses/by-nc/4.0/) which permits copy and redistribute the material just in noncommercial usages, provided the original work is properly cited. 
same clinical situation. Radiographs were performed after clinical evaluation and completion of the data collection form for each patient. Standard ankle and foot series were obtained if there was pain or tenderness in the malleolar or midfoot zones, respectively. Radiography results were interpreted by an orthopedic surgery resident who had not visited or examined the patients. For statistical analysis, SPSS 12.0 (SPSS Inc. Chicago, Illinois, the United States) was used. Sensitivity, specificity, positive and negative likelihood ratio, and positive and negative predictive value with a $95 \% \mathrm{CI}$ were calculated.

\section{Results}

We evaluated 140 patients (84 males and 56 females) with a mean age of 32.2 years (range, 8 - 76 years). The reported mechanism of injury by patients were fall in 58 , twisting in 52, and sustained Road Traffic Accidents (RTA) in 30 patients (Table 1 ). The fall was frequently occurred in home or workplace (71\%) with only $29 \%$ of cases being related to sports.

Of the 140 evaluated patients, 71 did not met criteria for radiologic evaluation, with no fracture. The remaining 69 presented with positive criteria (Table 2 ). Of these, 48 patients had fracture among which $34(70.83 \%)$ were in the malleolar zone and $14(29.16 \%)$ in the midfoot zone (Table 3).

As shown in Table 4, the Ottawa rules sensitivity in detecting fracture was $100 \%$ (89.6\%-100\%) for both malleolar and midfoot fractures. The negative predictive value was also $100 \%$. We estimated the sensitivity to be $100 \%$ and specificity to be $78.7 \%$. The positive predictive value was 71.7\%. The implementation of the OARs appears to have the potential to reduce the number of radiographs for the assessment of these patients by about $51 \%$.

\begin{tabular}{|c|c|}
\hline Variable & Value \\
\hline \multicolumn{2}{|l|}{ Gender $^{\mathrm{a}}$} \\
\hline Male & $84(60)$ \\
\hline Female & $56(40)$ \\
\hline Mean age, $y$ & 32.2 \\
\hline \multicolumn{2}{|l|}{ Mechanism of injury ${ }^{a}$} \\
\hline Fall & $58(41.4)$ \\
\hline Twisting & $52(37.1)$ \\
\hline Road Traffic accident & $30(21.4)$ \\
\hline
\end{tabular}

${ }^{\mathrm{a}}$ Data are presented as No. (\%).

Table 2. Results According to Criteria of Ottawa

\begin{tabular}{lcc} 
& Positive & Negative \\
\hline Fractures & 48 & 0 \\
Sprains & 21 & 71 \\
\hline
\end{tabular}

Table 3. Distribution of Fractures ${ }^{a}$

\begin{tabular}{lc}
\hline Variable & Value \\
\hline Fractures & $48(34.28)$ \\
Malleolar zone & $34(70.83)$ \\
\hline Lateral malleoli & $21(43.75)$ \\
Medial malleoli & $10(20.83)$ \\
Bimalleolar & $3(6.25)$ \\
Calcaneus & 0 \\
\hline Talus & 0 \\
Midfoot zone & $14(29.16)$ \\
Base of 5th metatarsal & $11(22.91)$ \\
Cuboid & $2(4.16)$ \\
\hline Navicular & $1(0.71)$ \\
\hline Cuneiforms & 0 \\
\hline a
\end{tabular}

a Data are presented as No. (\%).

\begin{tabular}{ll}
\hline \multicolumn{2}{l}{ Table 4. Statistical Results of the Study a } \\
\hline Variable & Value \\
\hline Sensitivity & 100 \\
Specificity & 78.7 \\
Negative predictive value & 100 \\
Positive predictive value & 71.6 \\
\hline
\end{tabular}

${ }^{\mathrm{a}}$ Data are presented as (\%).

\section{Discussion}

The evaluation of an acute ankle injury may seem to be simple. Only $15 \%$ of these patients have a fracture. The difficulty lies to differentiate which ones has fractures. Radiographs of the ankle and/or foot are frequently done in the ED, which proves to be unnecessary in majority of the cases. Several studies have been conducted to establish predictive rules for the use of radiography in ankle injuries (3-5). The OARs were created and first validated in 1990. It rapidly became an important tool in the emergency scenarios for its simplicity and efficacy, resulting in saving time and money by reducing the number of patients referred for the radiographs. Since its implementation, several studies have been conducted in the United States, the United Kingdom, France, Greece, Spain, Iran, and Australia that validated the rules (6-11). Bachmann et al. (12) performed a systematic review of 27 studies that assessed the implementation of these rules. The sensitivity was $100 \%$, with the possibility of reducing the number of radiographs by $30 \%$ to $40 \%$.

Without validation, however, even well-defined decision-making rules may not be suitable due to different patient population. Secondly, physicians may not be able to use the rule comprehensively or perfectly due to clinical setting and training differences. Because there is no 
study described for the validation of OARs in India, we felt the need to accomplish this, which demonstrate its applicability in the Indian population.

Our results were similar to other studies, revealing sensitivity and negative predictive value of $100 \%$. The great increase in the imaging examination has made the orthopedic surgeon increasingly dependent on the radiographs for diagnosis. The possible reduction of $51 \%$ in the radiographs means that the OARs protocol will allow doctors to spend more time examining the patients, reduce waiting time for the patients, and decrease the radiation hazard to both patients and emergency staff. In the present era of cost containment, increased awareness of unnecessary tests and procedures will only become more significant (13). Accordingly, clinicians will need to use these rules to cut cost for both the hospital and patient.

Despite the good results shown by several studies, the application of these criteria has some limitations, which are related to the pressure caused by the patients and their attendants to perform radiography. The current study had some limitations. The relatively low number of cases made it difficult to generalize the results to the whole population and the inter-observer reliability among the physician was not tested.

In conclusion, in patients with ankle injury and the presence of no positive OARs, there is no indication to perform radiography, since the probability of no fracture is $100 \%$. For our study, these criteria appear to have the potential to reduce the required number of radiographs by about $51 \%$. The results of this study show no false negatives and are in agreement with the results of other similar studies, which encourage us to implement these criteria in our services urgently, with all the socioeconomic implications resulting the reform.

\section{Authors' Contributions}

Both authors participated in all parts of the study.

\section{References}

1. Donken CC, Al-Khateeb H, Verhofstad MH, van Laarhoven CJ. Surgical versus conservative interventions for treating ankle fractures in adults. Cochrane Database Syst Rev. 2012;8:CD008470.

2. Yazdani S, Jahandideh H, Ghofrani H. Validation of the Ottawa Ankle Rules in Iran: a prospective survey. BMC Emerg Med. 2006;6:3.

3. Stiell IG, Greenberg GH, McKnight RD, Nair RC, McDowell I, Worthington JR. A study to develop clinical decision rules for the use of radiography in acute ankle injuries. Ann Emerg Med. 1992;21(4):384-90.

4. Sujitkumar P, Hadfield JM, Yates DW. Sprain or fracture? An analysis of 2000 ankle injuries. Arch Emerg Med.1986;3(2):101-6.

5. Kelly AM, Richards D, Kerr L, Grant J, O'Donovan P, Basire K, et al. Failed validation of a clinical decision rule for the use of radiography in acute ankle injury. N Z Med J.1994;107(982):294-5.

6. Hale WR. Can the Ottawa ankle decision rules be applied in the United States? West J Med.1996;164(4):363.

7. Broomhead A, Stuart P. Validation of the Ottawa Ankle Rules in Australia. Emerg Med (Fremantle). 2003;15(2):126-32.

8. Auleley GR, Kerboull L, Durieux P, Cosquer M, Courpied JP, Ravaud P. Validation of the Ottawa ankle rules in France: a study in the surgical emergency department of a teaching hospital. Ann Emerg Med.1998;32(1):14-8.

9. Auleley GR, Ravaud P, Giraudeau B, Kerboull L, Nizard R, Massin P, et al. Implementation of the Ottawa ankle rules in France. A multicenter randomized controlled trial. JAMA.1997;277(24):1935-9.

10. Papacostas E, Malliaropoulos N, Papadopoulos A, Liouliakis C. Validation of Ottawa ankle rules protocol in Greek athletes: study in the emergency departments of a district general hospital and a sports injuries clinic. BrJ Sports Med.2001;35(6):445-7.

11. Cuello-Garcia C, Ruiz-Flores A, Ramos-Gomez L, Medina-Lopez ME. [The Ottawa ankle rules: evaluation in a pediatric emergency setting in Mexico]. An Pediatr(Barc). 2004;60(5):454-8.

12. Bachmann LM, ter Riet G. The Ottawa rules for ankle sprains. Hosp Med. 2004;65(3):132-3.

13. Jenkin M, Sitler MR, Kelly JD. Clinical usefulness of the Ottawa Ankle Rules for detecting fractures of the ankle and midfoot. $J$ Athl Train. 2010;45(5):480-2. 